\title{
Rings in which Every Simple Right R-Module is Flat
}

Raida D. Mahmood

raida.1961@uomosul.edu.iq

Dept. Of Mathematics

College of Computer and Mathematical Science

University of Mosul, Iraq

Received on: 23/09/2002

Accepted on: 05/11/2002

\section{ABSTRACT}

The objective of this paper is to initiate the study of rings in which each simple right $\mathrm{R}$-module is flat, such rings will be called right SF-rings. Some important properties of right SFrings are obtained. Among other results we prove that: If $\mathrm{R}$ is a semi prime ERT right SF-ring with zero socle, then $R$ is a strongly regular ring.

Keywords: strongly regular rings, SF-ring, reduced rings.

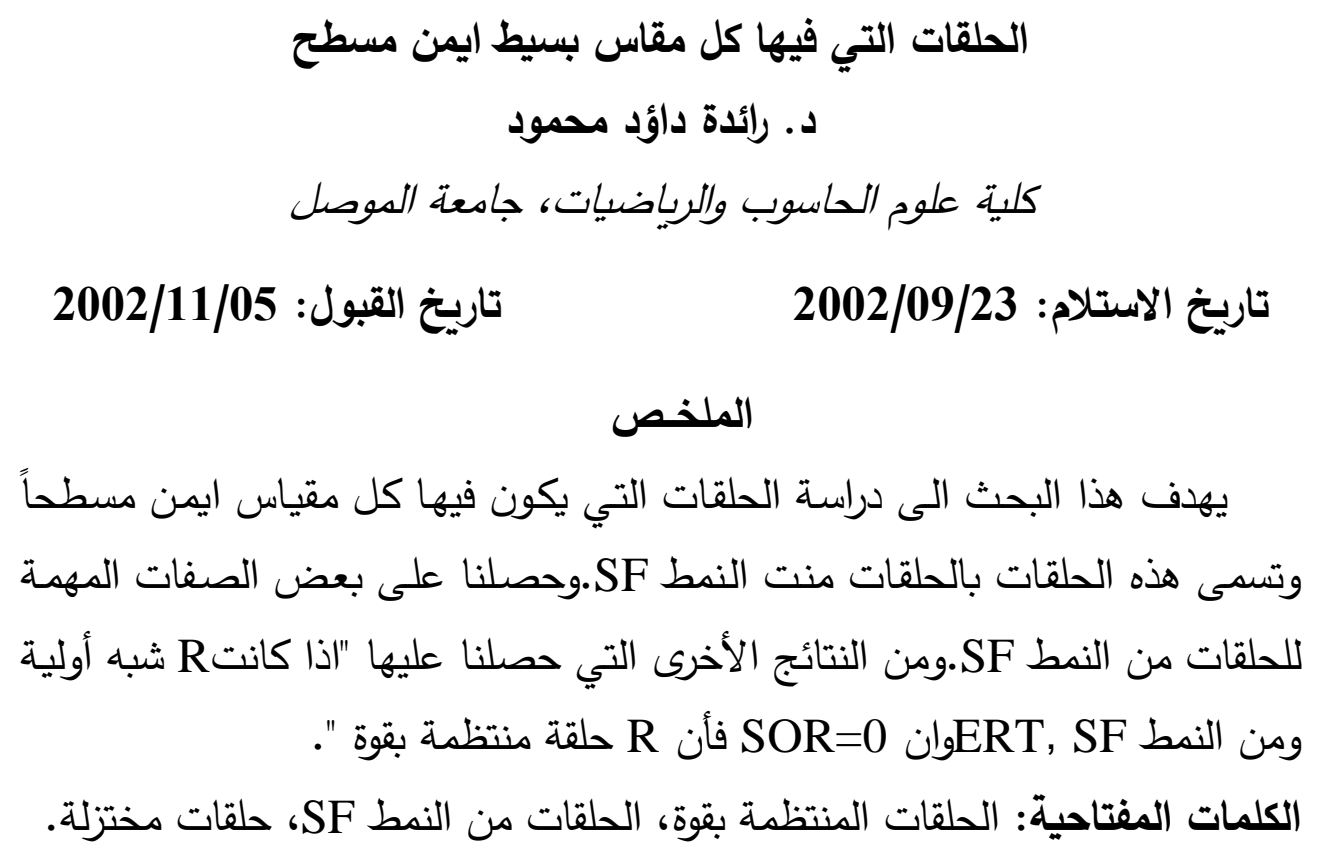




\section{Introduction:}

Throughout this paper, $\mathrm{R}$ denotes associative ring with identity and all modules are unitary. $\mathrm{J}(\mathrm{R})$ and $\mathrm{Y}(\mathrm{R})$ denote the Jacobson radical and the singular right ideal of $R$, respectively. For any nonempty subset $\mathrm{X}$ of a ring $\mathrm{R}$, the right (resp. left) annihilater of $X$ will hdbenoted by $r(X)$ (resp. 1(X)). Recall that:

(1) A ring $R$ is ERT if every essential right ideal of $R$ is a sided,[6]

(2) R is said to be von Nuumann regular (or just regular) if, $a \in a R a$,for every $a \in R$, and $R$ is called strongly regular if $a \in a^{2}$ R.

In [3] Ming asked the following question:

Is a semi prime right SF-ring, all of whose essential right ideals are two-sided von Neumann regular?

In this paper, we give conditions for a semi prime right SF-ring all of whose essential right ideals are two sided to be von Neumann regular.

\section{Basic Properties:}

Following [5], a ring $\mathrm{R}$ is called a right (left) SF-ring, if every simple right (left) $\mathrm{R}$ - module is flat.

The following lemma which is due to [7], plays a central role in several of our proofs: Lemma 2.1:

Let $\mathrm{I}$ be a right ideal of $\mathrm{R}$. Then $\mathrm{R} / \mathrm{I}$ is a flat $\mathrm{R}$-module if and only if for each ae I, there exists be I such that $a=b a$. 
We shall begin with the following result:

\section{Proposition 2.2:}

If $\mathrm{R}$ is a right SF-ring. Then

(1) Any reduced principal right ideal of $\mathrm{R}$ is a direct summand.

(2) Every left or right R-module is divisible.

\section{Proof (1):}

Let $\mathrm{I}=\mathrm{aR}$ be a reduced principal right ideal of $\mathrm{R}$ and let $\mathrm{aR}+\mathrm{r}(\mathrm{a})$ \# $\mathrm{R}$. Then there exists a maximal right ideal $\mathrm{M}$ of $\mathrm{R}$ containing $\mathrm{aR}+\mathrm{r}(\mathrm{a})$.

Now, since $R / M$ is flat, then $a=b a$, for some $b$ in $R$. Whence $\quad l-b \in l(a)=r(a) \subseteq M$. Yielding 1 e $M$ which contradicts $\mathrm{M} * \mathrm{R}$. In particular

$\operatorname{ar}+c=1$, for some re $R$ and $c \in r(a)$, whence $a^{2} r=a$. If we set $\mathrm{d}=\mathrm{ar}^{2} \in 1$, then $\mathrm{a}=\mathrm{a}^{2} \mathrm{~d}$. Clearly, $(\mathrm{a}-\mathrm{ada})^{2}=0$ implies $\mathrm{a}=\mathrm{ada}$ and hence $\mathrm{I}=\mathrm{eR}$, where $\mathrm{e}=\mathrm{ad}$, is idempotent element. Thus I is a direct summand.

\section{Proof (2):}

It is sufficient to prove that any non-zero divisor $\mathrm{c}$ of $\mathrm{R}$ is invertible. For then, if $\mathrm{dc}=\mathrm{cd}=1$, any right $\mathrm{R}$-module $\mathrm{M}$ satisfies $\mathrm{M}=\mathrm{Mdc} \subseteq \mathrm{Mc} \subseteq \mathrm{M}$, whence $\mathrm{M}=\mathrm{Mc}$ (similarly, any left $\mathrm{R}$-module is divisible). Suppose that $\mathrm{c} R \neq \mathrm{R}$. Let $\mathrm{K}$ be a maximal right ideal containing $c R$. Since $R / K$ is flat, there exist ueK, such 
that $c=u c$. Now, $r(c)=1(c)=0$ implies $u=1$, contradicting $K \neq R$. This proves that $\mathrm{cd}=1$ for some $\mathrm{de} \mathrm{R}$ and hence $\mathrm{dc}=1$.

\section{Proposition 2.3:}

Let $\mathrm{R}$ be a right SF-ring. Then either $r(M)=0$ or $M$ is a direct summand.

\section{Proof:}

Suppose that $r(M) \neq 0$ and let $b \in M \cap r(M)$. Then $b \in M$ and $M b=0$. Since $R / M$ is flat then there exists $a \in M$ such that $b=a b$. Now $b=a b \in M b=0$, so $b=0$. Thus $M \cap r(M)=0$, this means that $\mathrm{M}$ not can be essential and hence $\mathrm{M}$ is a direct summand. Therefore $\mathrm{r}(\mathrm{M}) \oplus \mathrm{M}=\mathrm{R}$.

\section{The Connection Between SF-Rings and Other Rings:}

In this section we study the connection between SF-rings, biregular rings and strongly regular rings.

Recall that the right (left) socle of a ring $\mathrm{R}$ is defined to be the sum of all minimal right (left) ideals of $\mathrm{R}$. It is well know that in a semi prime ring $\mathrm{R}$, the right and left socles of $\mathrm{R}$ coincide, which will be denoted by socR[8].

Following [4], a ring $\mathrm{R}$ is biregular if $\mathrm{RaR}$ is generated by a central idempotent for each $\mathrm{a} \in \mathrm{R}$. 


\section{Theorem 3.1:}

Let $\mathrm{R}$ be an ERT SF-ring with right zero socle and for every $a \in R, R a R$ is a principal right of $R$. Then $R$ is biregular.

Proof:

For any $a \in R$, set $M=R a R+1(R a R)$. Since $M$ is a maximal right ideal, then $M$ is a direct summand or essential. If $M$ is a direct summand of $\mathrm{R}$, then its complement is a minimal right ideal. This implies that $\mathrm{R}$ has a no-zero socle, which is a contradiction. So every maximal right ideal is essential and hence two-sided. By hypothesis $\mathrm{R} / \mathrm{M}$ is flat. Also $\mathrm{RaR}=\mathrm{bR}$ for some $b \in R$. Since $b \in M, b=d b$ for some $d \in M$. Then 1$\mathrm{d} \in \mathrm{l}(\mathrm{b}) \mathrm{cM}$ which yields $l \in \mathrm{M}$. Therefore $\mathrm{l}=\mathrm{bc}+\mathrm{v}, \mathrm{c} \in \mathrm{R}, \mathrm{v} \in \mathrm{l}(\mathrm{b})$ this implies that $b=b c b$. Therefore $R a R=b R=e R$ where $e=b c$ is idempotent. $\mathrm{R}$ is therefore semi-prime and hence $\mathrm{e}$ is central in $\mathrm{R}$. Thus $\mathrm{R}$ is biregular.

\section{Theorem 3.2:}

If $R$ is a reduced ring and every maximal right ideal of $R$ is either a right annihilator or flat, then $\mathrm{R}$ is strongly regular.

\section{Proof:}


Let $b \in R$. we claim first $b R+r(b)=R$. If not, there exists $a$ maximal right ideal $\mathrm{L}$ containing $\mathrm{bR}+\mathrm{r}(\mathrm{b})$. In case $\mathrm{R} / \mathrm{L}$ is flat, since $b \in L$, there exists $c \in L$ such that $b=c b$. Then $1-c \in l(b)=r(b) c L$, whence it follows $1 \in \mathrm{L}$, a

contradiction. On the other hand, in case $\mathrm{L}=\mathrm{r}(\mathrm{t})$ with some $0 \neq t \in R$, we have $\mathrm{t} \in !(b R+r(b)) \subseteq l(b)=r(b) \subseteq L=r(t)$. Then $t^{2}=0$, a contradiction. Therefore let $b R+r(b)=R$, and hence $R$, is strongly regular.

Now, we give under what condition the answer of the question of ring is affirmative.

\section{Proposition 3.2:}

Let $\mathrm{R}$ be a semi-prime ERT right SF-ring with zero socle. Then $\mathrm{R}$ is strongly regular.

\section{Proof;}

Let $\mathrm{M}$ be a maximal right ideal of $\mathrm{R}$. Then $\mathrm{M}$ is either a direct summand of $\mathrm{R}$ or an essential right ideal of $\mathrm{R}$. If $\mathrm{M}$ is a direct summand of $\mathrm{R}$, then its complement is a minimal right ideal. This implies that $\mathrm{R}$ has a nonzero socle, which is a contradiction. So every maximal right ideal is essential and hence two-sided.

Since R is semi-prime ERT, by applying [2,Lemma 2.1], we see that $R$ is right non-singular, and $J(R)=0$. So $R$ is isomorphic to a sub direct sum of division rings, which implies that $\mathrm{R}$ has no 
non-zero nilpotent elements. Therefore $\mathrm{R}$ is strongly regular.f 1 , proposition 1].

\section{Theorem 3.3:}

Let $\mathrm{R}$ be a prime ERT and right SF-ring. Then $\mathrm{R}$ has nonzero socle.

\section{Proof:}

Let $\mathrm{R}$ be a prime ERT and right SF-ring. If $\mathrm{SocR}=0$. By the above Theorem (3.2), $\mathrm{R}$ is strongly regular. Hence $\mathrm{R}$ is a division ring, and $\mathrm{Soc} R=\mathrm{R}$ contradicting our assumption. Therefore $\operatorname{Soc} R \neq(0)$. 


\section{REFERENCES}

(1)R.Y.C. Ming, (1974), " On Von Neumann regular rings ", Proc. Edinburgh Math. Soc. 19, 89-91.

(2)R.Y.C. Ming, (1978), "On Von Neumann regular rings, III". Monatsh. Math. 86, PP. 251-257.

(3) R.Y.C. Ming, (1980), "On Von Neumann regular rings". V, Math. J. Okayama Univ. No. 22, PP. 151-160.

(4) R.Y.C. Ming, (1983), " Maximal ideals in regular rings", Hokkaido Math. J. Vol. 12,PP: 119-128.

(5) R.Y.C. Ming, (1995), "A note on regular rings, II", Bull. Math. Soc. Sc. Math. Roumanie Tome 38 (86). PP. 169-173.

(6)R.Y.C. Ming, (1995), "A note on regular rings, III", Riv. Math. Univ. Parma, 6(1), PP 71-80.

(7)M.B. Rega, (1986), "On Von Neumann regular rings and SF-rings", Math. Japonica. 31, No. 6, PP.927-936.

(8)S.K. Jain, S.H. Mohamed and Surjeet Singh, (1969), Rings in which every right ideal is quasi-injective", Pacific. J. of Math. Vol. 31, No. 1, pp. 73-79. 